\title{
A Buffer Overhead Minimization Method for Multicast-based Handoff in Picocellular Networks
}

\author{
Eunyong Ha, Yanghee Choi, Chongsang Kim \\ Seoul National University \\ Department of Computer Engineering, Seoul National University, \\ Silim-Dong, Kwanak-Ku, Seoul 151-742, Korea \\ Telephone : +82-2-887-8992 \\ email : eyha@twins.snu.ac.kr, yhchoi@smart.snu.ac.kr, \\ cskim@sparc.snu.ac.kr
}

\begin{abstract}
A picocellular network consists of picocells whose radius is of the order of ten meters. The smaller cell size causes frequent handoffs of mobile hosts. In order to support seamless communication service to mobile users, we need a new handoff scheme. In this paper, we propose a multicast-based pre-handoff scheme, called SGMH ( SubGroup Multicastbased Handoff), which minimizes buffer overhead for seamless comnunication service as well as handoff processing delay. We show the performance in terms of blocking probability through analysis and simulation.
\end{abstract}

Keywords

Handoff, Buffer Overhead, SGMH, Picocellular Network, Multicast

\section{INTRODUCTION}

For the next generation of mobile communication systems an ubiquitous coverage is required. It is widely accepted that the coverage of places with high user densities can only be achieved with small cells(microcell and picocell). Picocells permit higher reuse of channels resulting in an increase in system capacity by several orders of magnitude. The smaller cell size in microcellular or picocellular systems, however, increases the number of handoffs (Nanda,1993). This implies that the time between handoffs and the time to complete handoffs becomes shorter. Thus in order to provide good quality of connection to mobile user, a faster handoff or pre-handoff method is needed.

In a two-tier system of microcells overlaid with macrocells, by assigning low mobility users to microcells and high mobility users to macrocells, it decreases the total number of handoffs (Yeung,1995)(Chih,1993).

In (Acampora,1994), (Yu,1995) and (Ghai,1994), they construct a multicast tree for handoffs in the near future and when a handoff occurs, the reserved connections are used. They drop the handoff processing delay but consume lots of resources.

In this paper, we propose a multicast-based pre-handoff scheme, called SGMH (Sub- 


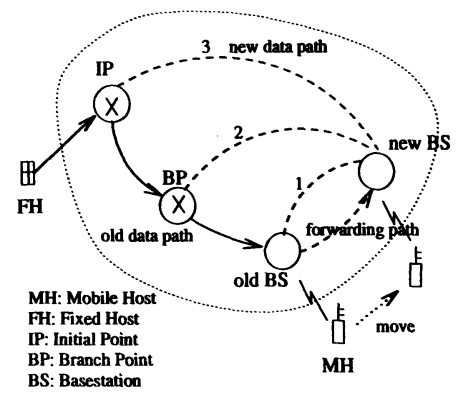

Figure 1 Point-to-point handoffs

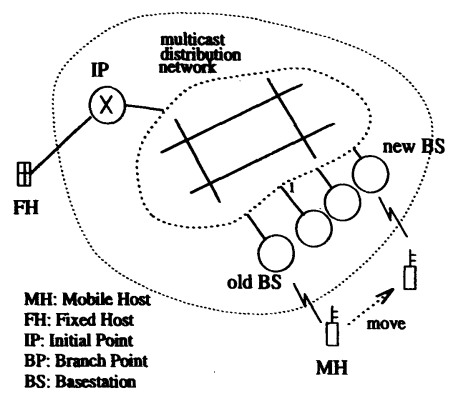

Figure 2 Multicast-based handoffs

Group Multicast-based Handoff), for providing mobile user with seamless connection service. SGMH can not only minimize the buffer overhead of multicast-based handoff methods but also provides seamless connection service to mobile user.

This paper is organized as follows. In section 2, we will survey about various handoff methods in the literature. In section 3, we propose SGMH method and explain about SGMH in detail: the concept of SGMH, an architecture of picocellular networks, method of subgroup selection, the traffic model of MH's mobility and the handoff blocking probability. Finally, in section 4, we study on the relationship between the reduction of buffer overhead and the blocking probability through simulations.

\section{VARIOUS HANDOFF METHODS}

Generally handoff methods presented in the literature can be divided into two categories : pont-to-point and point-to-multipoint. As in Figure 1, point-to-point handoff methods can be divided into three categories: path extension, forward-and-reroute and anchorbased handoff methods. (1) In case of path extension method (label 1) (Keeton,1993), when a MH moves into a new cell, it informs the new BS of its old BS information and its connection information. The new BS sends a path extension request message to the old BS. The old BS extends the MH's connection to the new BS. After connection extension, the old BS resumes sending data to the MH through the newly extended path. The problems of this method are as follows: the path length becomes very long and path cycle may happen. Therefore a path optimization algorithm and special cycle elimination algorithm are required. (2) In the forward-and-reroute method (label 2) (Keeton,1993), after a MH greets to the new BS, the new BS sends a forward-data-request message to the old BS. The old BS setups a forwarding path to the new BS and forwards data to the MH. And then the new BS searches for a branch point which is closest to the new BS and reroutes the connection to the branch point. But they are time consuming jobs. (3) The anchor-based handoff (label 3) (Akyildiz,1996), is similar to the forward-and-reroute method with difference that the forward point is fixed at the initial BS chosen at the call setup. It has also the problem that the path length between the initial BS and the new BS may be long.

Multicast-based handoff methods can be subdivided into two categories according to the method of constructing a multicast distribution tree. (1) Static: a multicast tree is created at call setup. The multicast tree does not change for the call lifetime (Acampora,1994). (2) Dynamic: a multicast tree changes dynamically during the call lifetime according to the MH's mobility (Ghai,1994). In multicast-based handoffs, it is before handoff that 
a multicast tree is composed of neighboring basestations and data is multicasted to all member BSs. When the MH enters a new cell, it greets the new BS and receives data from the new BS immediately. And the new BS informs the RS of the MH's location change. Therefore there is no service break. But they cost lots of buffer space at each member BS.

In summary, point-to-point handoff methods are not fit for seamless connection service in picocellular networks because of their inherent characteristics of data forwarding and rerouting overhead. Multicast-based handoff methods are appropriate for the seamless service because there is no handoff delay. But they have a main drawback of large buffer overhead because the preallocated buffers in member BSs excepts for the current BS are not used for the dwell time of the $\mathrm{MH}$ in the current cell. So we propose a multicat-based handoff method which can minimize buffer overhead.

\section{THE PROPOSED SGMH HANDOFF METHOD}

In this section, we will explain about the proposed SGMH handoff method in details : a picocellular network architecture, the concept of SGMH, and the selection of multicast subgroups.

\subsection{A picocellular network architecture}

We assume that a picocellular network, as shown in Figure 3, is composed of three layers: mobile host layer at the bottom, basestation layer in the middle and region server layer at the top. As shown in Figure 3, MH reports the received signal strengths to RS periodically. BS plays a role of mobile network access point and manages radio channels and buffer space for seamless connection service. RS covers one service area and performs connection-related functions: selection of multicast subgroups, setup multicast tree and data multicasting and so on. For seamless connection service, each layer must maintain connection-related information. We will not describe about the information here.

Compared with conventional cellular networks, picocellular network has following features: small cell size, high rate of frequency reuse, small-sized handset with low power consumption, high throughput, frequent handoffs and so on. Particularly frequent handoff is a main drawback. For example, if a mobile user moves with the average speed of 2 meters per second and picocell's diameter is 10 meters, handoff happens every 5 seconds. The mobile user experiences frequent service breaks. Therefore, for seamless connection service, we need a new handoff method fit for picocellular networks.

\subsection{Concept}

Here we will explain about the concept of the proposed SGMH method. The smaller cell size causes frequent handoffs in a picocellular network (Nanda,1993). The processing delay of frequent handoffs is an obstacle to provide mobile user with seamless service. But if the mobile system performs necessary jobs before the handoff, such as connectin pre-setup to the candidate BSs and transfer of data to the BSs, it can support mobile user with the seamless service.

In SGMH, RS predicts the MH's mobility and chooses the multicast group of the MH based on the velocity of the MH and the distances from the MH's current position to candidate basestations and sends data to member BSs before handoff. When the MH enters a new member BS, the MH can receive data from the new BS immediately. Therefore, SGMH method can eliminate handoff delays : the time to forward the transit data to the $\mathrm{MH}$, the time to reroute the connection to the new BS and the time to resume sending 


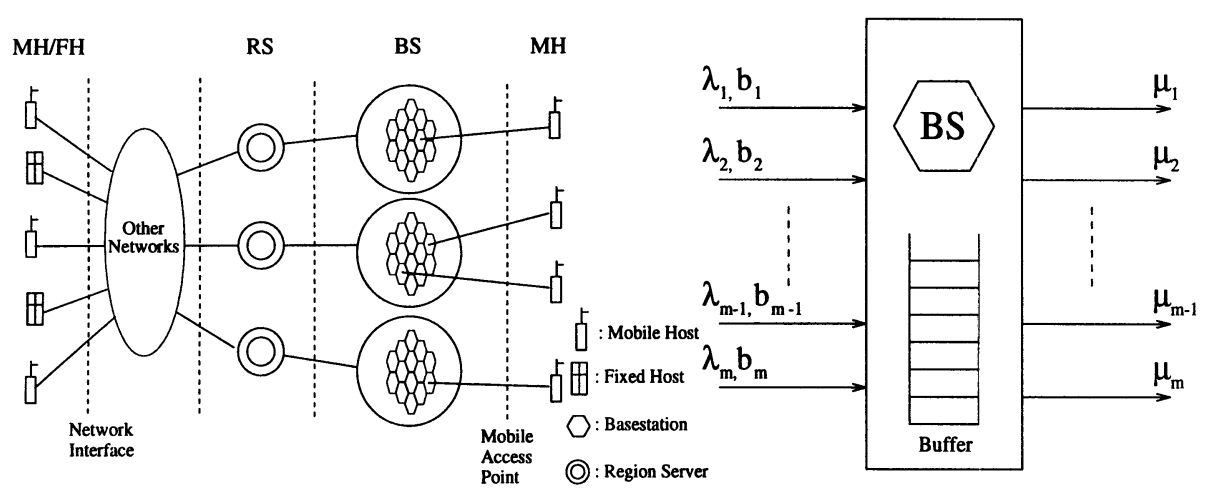

Figure 3 An architeture of picocellular network

Figure 4 Traffic model of a BS

data. In addition to the elimination of the handoff delay, becaue each member BS stores differnt amount of data according to its distance from the MH, SGMH can minimize the total buffer overhead and lowers the blocking probability of handoff calls and newly arriving calls due to the shortage of buffer space.

\subsection{Selection of multicast subgroups}

In order to estimate the location and the speed of a mobile user in a cellular system, it is reasonable to use the information about field strength and distance from the BS, both of which are supplied by the system. Various methods to obtain this information are broken down into three basic categories: radiolocation, dead-reckoning, and proximity methods (Kennenmann,1995). In order to estimate the position of the $\mathrm{MH}$, they in general use trilateration and require installation of additional devices in the $\mathrm{MH}$ and the $\mathrm{BS}$.

Here we do not consider the location methods, but we use the porposed methods to select the multicast subgroups of the MH. As shown in Figure 3, RS serves one service area and maintains the tables on the information about the cell layout and the signal propagation model. RS chooses the subgroups of the $\mathrm{MH}$ based on those informations. The procedure is as following. The $\mathrm{MH}$ informs the $\mathrm{RS}$ of the signal strengths received from neighboring BSs periodically. By using this information, RS selects the candidate BSs which the MH may move into in the near future. RS divides the BSs into several subgroups according to the expected handoff times. Because the earlier selection influences on the network performance, the selection time, if possible, is delayed to the latest time that the delay does not effect on the seamless connection service.

Let us introduce several variables. $T_{s g}$ denotes the time taken to select the candidate basestations and divide them into several subgroups. $T_{\text {join }}$ represents the sum of the time taken to setup a multicast distribution tree and the longest time among the joining times of member BSs: i.e., $T_{\text {join }}=\operatorname{Max}\left(T_{\text {join }}(i)\right.$ for each member BS $)$. Then the total time taken to setup the subgroups of the $\mathrm{MH}$ is equal to $T_{g s}=T_{s g}+T_{\text {join }}$. Therefore, the group selection can start at the time which is calculated by subtracting the group selectin time $T_{g s}$ from the expected handoff time $T_{h o}$ : i.e., $T_{h o}-T_{g s}$. In case that there is not enough time to setup the subgroups, SGMH can not provide MHs with seamless service and thus the conventional handoff method is used. 


\subsection{Computation of buffer overhead}

We define several parameters for calculating buffer overhead of SGMH.

- $G$ : the number of member BSs in a multicast group

- $m$ : the number of subgroups classified by their distances from the MH's current position

- $S G_{i}$ : the number of BSs of the $i$ th subgroup

- $V$ : the estimated speed of the $\mathrm{MH}$

- $D_{i}:$ the distance from member $\mathrm{BS}_{i}$ to the $\mathrm{MH}$

- $T_{d}$ : the real dwell time of the MH in the current cell

- $T_{p_{i}}:$ the expected arrival time of the $\mathrm{MH}$ at $\mathrm{BS}_{i}$

- $N^{\prime}$ : the number of packets arrived at current cell during $T_{d}$

- $K_{i}$ : the number of packets arrived at a BS of the $i$ th subgroup between $T_{p_{i}}$ and $T_{d}$

The expected arrival time of the $\mathrm{MH}$ at a member $\mathrm{BS}_{i}, T_{p_{i}}$, is calculated by dividing the distance $D_{i}$ by the speed $V$ : i.e., $T_{p_{i}}=D_{i} / V$.

The buffer overhead exists only if $T_{p_{i}}<T_{d}$, because for seamless connection service other BSs except for the current BS must store packets arrived at between the expected handoff time $T_{p_{i}}$ and the real sojourn time $T_{d}$. In case of the wrong expectation $T_{p_{i}}>T_{d}$, there is no buffer overhead. Thus, the buffer overhead of SGMH is equal to $B=\sum_{i=1}^{m} K_{i} \times S G_{i}$.

Let us take an example that the multicast group of the $M H$ is composed of $G=\{a, b 1$, b2, b3, c1, c2, c3, d2 \}.

(1) In case of the simple multicast-based handoff in (Keeton,1993), the packets to the MH are multicasted to all member BSs and are stored in buffer space for the near future usage. Thus, the total buffer overhead $B$ is equal to the product of the multicast group size and the number of packets transmitted for the real dwell time : i.e., $B=N \times(|G|-1)=$ $7 \times N$.

(2) In case of the method proposed in (Ghai,1994), the buffer overhead exists only if the expected handoff time is before the real handoff time : i.e., $T_{p}<T_{d}$. All member BSs except for the current BS must store packets transmitted for the time interval between the expected time $T_{p}$ and the real handoff time $T_{d}$. The buffer overhead is $B=(|G|-1) \times K=$ $7 \times K$.

(3) In SGMH, the multicast group is divided into several subgroups according to the distances of BSs from the current MH's location. If the multicast group $G$ is divided into $\mathrm{G}=\{\{\mathrm{a}\},\{\mathrm{b} 1, \mathrm{~b} 2, \mathrm{~b} 3\},\{\mathrm{c} 1, \mathrm{c} 2, \mathrm{c} 3\},\{\mathrm{d} 2\}\}$, in case of $T_{p_{i}}<T_{d}$, the total buffer overhead is equal to $B=3 \times K_{2}+3 \times K_{3}+1 \times K_{4}$ where $K_{1}>K_{2}>K_{3}$.

Therefore SGMH method has the lowest buffer overhead among three multicast-based handoff methods.

\subsection{Analytic model}

We consider a picocellular network as shown in Figure 3 with a suffciently large number of cells with the same geometric shape. Each cell is surrounded by the same number of neighboring cells. Each BS can belong to many subgroups and for the seamless connection service, the BS must reserve buffer space per each subgroup request. Therefore, the BS can be modeled as shown in Figure 4 .

It is assumed that the total buffer capacity available to a BS is denoted by $C$ and the buffer requests are divided into $m$ classes and each class requires different amount of buffer units. And the buffer request arrivals follow a Poisson process and the buffer holding times are distributed exponentially.

We define several parameters for each class $i=1,2,3, \ldots, m$ as followings: 
- $\lambda_{i}$ : the request arrival rate

- $1 / \mu_{i}$ : the mean buffer holding time

- $b_{i}$ : the number of required buffer units

- $P_{B_{i}}$ : blocking probability

- $P_{B_{i}, \text { max }}$ : maximum permissible blocking probability

- $n=\left(n_{1}, n_{2}, n_{3}, n_{4}, \ldots, n_{m}\right):$ the current state of a BS denoted by the number of requests of each class in progress

The number of busy buffer units is given by $n \times b=\sum_{i=1}^{m} n_{i} \times b_{i}$. The problem is to provide each class with blocking probabilities less than or equal to maximum permissible blocking probabilities.

Now let us compute the blocking probability of a handoff request. The blocking probability is dependent on the strategy allocating the buffer space. The allocation policies can be divided into three categories: complete sharing, fixed allocation and hybrid allocation (see (Tekinay,1993) for more details).

Here we consider only the blocking probabilities under the complete sharing policy because the key issue of this paper is not the buffer allocation methods but the evaluation of the proposed SGMH method.

From the viewpoint of each BS, the traffic of buffer requests can be divided into $m$ classes. The BS, therefore, is considered as a heterogeneous system and can be modeled as an $m$-dimensional Markov Chain. The transition probabilities along the $i$ th axis are dependent on the arrival rate and the service rate of requests of the $i$ th subgroup: i.e., $P_{n, n+1}=\lambda_{i} \cdot \Delta t$ and $P_{n, n-1}=\mu_{i} \cdot \Delta t$. The blocking probability of the $i$ th subgroup is the sum of the probabilities of the blocking states of the $i$ th subgroup. A blocking state of the $i$ th subgroup is the state from which a transition to a higher state along the $i$ th axis is not allowed. For example, if the state is $n=\left(n_{1}, n_{2}, n_{3}, \ldots, n_{i}, \ldots, n_{m}\right)$ and the next higher state $n=\left(n_{1}, n_{2}, n_{3}, \ldots, n_{i}+1, \ldots, n_{m}\right)$ is not allowed, the current state is a blocking state.

The traffic of the $i$ th subgroup in a BS can be modeled as $M / M / k / C$ Erlang's loss system. The probability of having $k$ calls of the $i$ th subgroup is equal to Equation(1).

$P_{k}=\frac{\left(\lambda_{i} / \mu_{i}\right)^{k}}{k !} \times P_{0}$

And the probability of the current state of the BS, $n=\left(n_{1}, n_{2}, n_{3}, \ldots, n_{i}, \ldots, n_{m}\right)$, is obtained as Equation(2).

$P(n)=P(0) \times \prod_{i=1}^{m} \frac{\left(\lambda_{i} / \mu_{i}\right)^{n_{i}}}{n_{i} !}$

Therefore, the probability of a request of the $i$ th subgroup becomes to Equation(3) where $B_{i}$ is the set of blocking states for $i$ th subgroup.

$P_{B_{i}}=\sum_{n \in B_{i}} P(n)$

\section{SIMULATION RESULT}

Here we will investigate how much the reduction of buffer overhead of SGMH method has an effect on lowering the blocking probability of the handoff calls and the newly arriving calls through simulations. 
Simulation runs in two steps. Firstly, we search for the total buffer capacity $C$ which is sufficient to satisfy for the given maximum permissible blocking probabilities of each subgroup traffic requests. And then for the selected buffer capacity, we measure the blocking probability with the variation of traffic intensity.

It is assumed that the number of subgroups is two. The simulation parameters in Table 1 are followings: $C$ denotes the total buffer capacity of each BS, $B$ means the requested buffer units of each subgroup handoff, $1 / \lambda$ means the mean arrival time and $1 / \mu$ means the mean buffer holding time respectively. And the arrival times of handoff calls and the buffer holding times are distribued exponetially. The values of parameters in Table 1 are not real values but relative values.

The first two simulation sets describe the cases that the movement of MHs is affected by the surroundings such as city streets and buildings. They are different by the expectation accuracy of the handoff time; the first one is more accurate than the second one. Figure 5 shows that the increase of buffer capacity of the BS lowers the blocking probabilities of each subgroup handoff calls and the blocking probability of the second subgroup calls is lower than that of the first subgroup calls. Figure 5 shows that the total buffer capacity with the maximum permissible blocking probability of less than $5 \%$ is about 20 buffer units.

Figure 7 and Figure 8 show the blocking probability with the variation of the traffic intensity of each subgroup calls. The blocking probability of the first set is lower than the second set because it requires less buffer units than the second set. The simulation results show that the blocking probability of SGMH method is much lower than that of the simple multicast-based handoff method.

And the last two simulation sets have different subgroup sizes, that is, they describe the environment that the mobility of the $\mathrm{MH}$ is not affected by the surroundings. In these cases, the arrival rate of the first subgroup calls is higher than that of the second subgrouop calls. As in Figure 6, we choose the total buffer capacity of 30 buffer units. Figure 9 and Figure 10 show the blocking probability of the first subgroup calls and the second subgroup calls for the simulation set 3 and set 4 repectively. They shows that SGMH is better than simple multicast-based handoff in the blocking probability. And the simulation set 3 with the high expectation accuracy also has better performance than the simulation set 4 .

In summary, the proposed SGMH method reduces the total buffer overhead and owing to the buffer reduction the blocking probability of handoff calls also decreases.

\section{CONCLUSION}

In this paper we proposed a multicast-based handoff method, so-called SGMH, which minimizes the service disruption delay owing to the frequent handoffs in the picocellular networks with the cell size of the order of ten meters or more. Because SGMH method takes consideration on the MH's spending time before the handoff and the distance from the MH's current location to a candidate handoff BS, it eliminates the handoff delay and reduces the buffer overhead significantly. And SGMH method also has the effect on dropping the handoff blocking probability. We evaluated the performance of SGMH method through the analysis and the simulation in terms of blocking probability and compared with the multicast-based handoff method. The simulation result shows that SGMH method is superior to the multicast-based handoff method. In the future, we will analyze the proposed SGMH method in various performance measures and design the protocol of SGMH method. 
Table 1 Simulation parameters

\begin{tabular}{ccccccccccccccc}
\hline & & \multicolumn{4}{c}{ Multicast Method } & \multicolumn{4}{c}{ SGMH Method } \\
\cline { 3 - 15 } Set & C & \multicolumn{3}{c}{ Subgroup 1 } & \multicolumn{3}{c}{ Subgroup 2 } & \multicolumn{3}{c}{ Subgroup 1 } & \multicolumn{3}{c}{ Subgroup 2 } \\
\cline { 3 - 14 } & & $\mathrm{B}$ & $1 / \lambda$ & $1 / \mu$ & $\mathrm{B}$ & $1 / \lambda$ & $1 / \mu$ & $\mathrm{B}$ & $1 / \lambda$ & $1 / \mu$ & $\mathrm{B}$ & $1 / \lambda$ & $1 / \mu$ \\
\hline 1 & 20 & 3 & 2 & 5 & 3 & 2 & 5 & 3 & 2 & 5 & 1 & 2 & 5 \\
\hline 2 & 20 & 3 & 2 & 5 & 3 & 2 & 5 & 3 & 2 & 5 & 2 & 2 & 5 \\
\hline 3 & 30 & 3 & 1 & 5 & 3 & 2 & 5 & 3 & 1 & 5 & 1 & 2 & 5 \\
\hline 4 & 30 & 3 & 1 & 5 & 3 & 2 & 5 & 3 & 1 & 5 & 2 & 2 & 5 \\
\hline
\end{tabular}

C: Total buffer capacity of BS

B: Requested buffer capacity

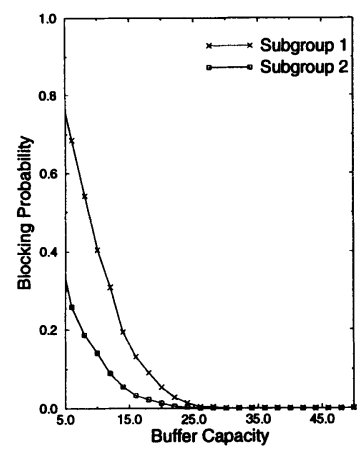

Figure 5 Set 1: Blk Prob vs Buffer Capacity

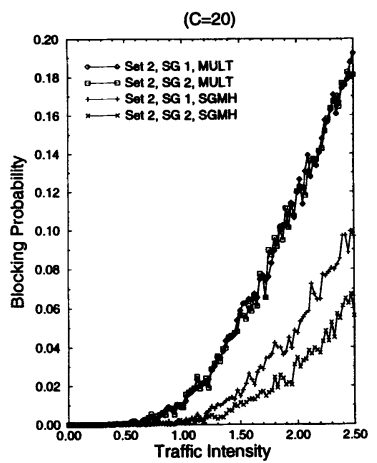

Figure 8 Set 2: Blk Prob vs Traffic Intensity

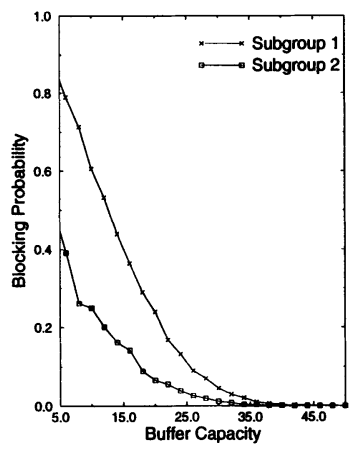

Figure 6 Set 3: Blk Prob vs Buffer Capacity

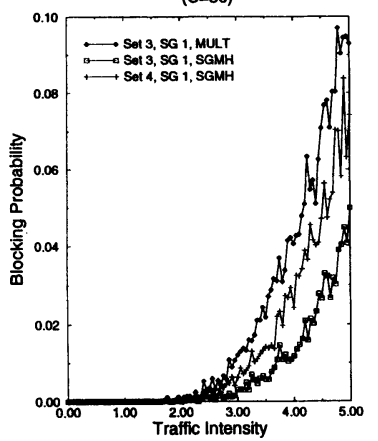

Figure 9 Subgroup 1 of Set 3,4 : Blk Prob vs Traffic Intensity

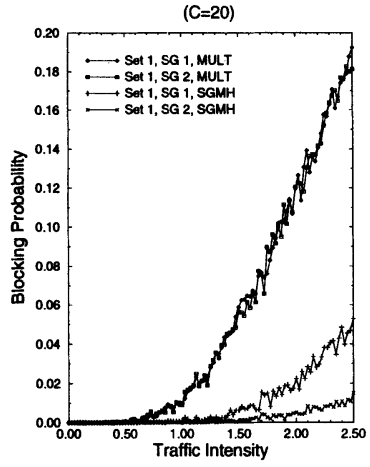

Figure 7 Set 1: Blk Prob vs Traffic Intensity

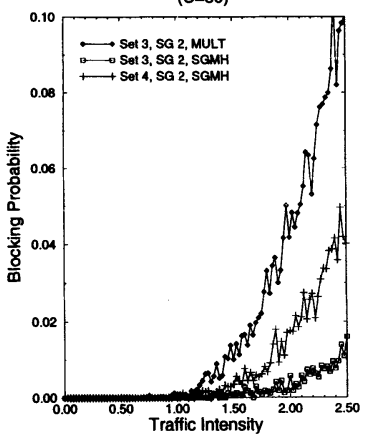

Figure 10 Subgroup 2 of Set 3,4: Blk Prob vs Traffic Intensity 


\section{REFERENCES}

Acampora, A.S. and Naghshineh, M. (1994) An Architecture and Methodology for MobileExecuted Handoff in Cellular ATM Networks. Journal on Selected Areas in Communications, 12(8),1365-1375, Oct.

Akyildiz, I.F., Ho, J.S.M. and Ulema, M. (1996) Performance Analysis of the Anchor Radio System Handover Method for Personal Access Communications System. In Proceedings of IEEE INFOCOM' '96, pages 1397-1404.

Ghai, R. and Singh, S. (1994) An Architecture and Communication Protocol for Picocellular Networks. IEEE Personal Communications, pages 36-46, Third Quater.

Chih-Lin I, Greenstein, L.J. and Gitlin, R.D. (1993) A Microcell/Macrocell Cellular Architecture for Low- and High-Mobility Wireless Users. Journal on Selected Areas in Communications, 11(6):885-891, Aug.

Keeton, K., Mah, B.A., Sehan, S., Karz, R.H. and Ferrari, D. (1993) Providing Connection-oriented Network Services to Mobile Hosts. In Proceedings of the USENIX Symposium, Aug.

Kennenmann, O. (1995) Locating Mobiles in Non-Flowing Traffic. In Proceedings of PIMRC'95, pages 274-278.

Nanda, S. (1993) Teletraffic models for urban and suburban microcells: Cell sizes and handoff rates. IEEE Transaction on Vehicular Technology, Nov.

Tekinay, S., Jabbari, B. and Kakaes, A. (1993) Modeling of Cellular Communication Networks with Hetrogeneous Traffic Sources. In Proceedings of ICUPC'93, pages 249253.

Yeung, K.L. and Nanda, S. (1995) Optimal Mobile-Determined Micro-Macro Cell Selection. In Proceedings of PIMRC'95, pages 294-299.

Yu, O.T.W. and Leung, V.C.M. (1995) B-ISDN Architectures and Protocols to Support Wireless Personal Communications Internetworking. In Proceedings of PIMRC'95, pages $768-772$.

Eunyong Ha received the B.S. and M.S. degrees in computer engineering from Seoul National University, Seoul, Korea, in 1986 and 1988 respectively. $\mathrm{He}$ is a $\mathrm{Ph} . \mathrm{D}$. student of Department of Computer Engineering at Seoul National University. His current research interests include wireless/mobile communication networks and multimedia systems.

Yanghee Choi received B.S. in electronics engineering from Seoul National University, M.S. in electrical engineering from KAIST, and Doctor of Engineering in Computer Science from ENST, Paris, in 1975, 1977 and 1984 respectively. He is an associate professor at the Department of Computer Engineering at Seoul National University. He has been with ETRI during 1977-1991. He is also associate director of the University Computing Center. He is associate editor for the Journal of Korea Information Science Society, and chairman of the Special Interest Group on Information Networking. His research interest lies in the field of multimedia systems and high-speed networking.

Chongsang Kim received the Ph.D degree in Electronic Engineering from Seoul National University. He is a professor at the Department of Computer Engineering at Seoul National University, where he has been since 1977. Dr. Kim was the president of the Korea Information Science Society from 1986 to 1988. He was also the chairman of the IEEE Korea Council during 1995. and director of the Research Institute of Advanced Computer Technology from 1993 to 1994 . His interests are computer architecture and computer networks. 